\title{
Examen d'un Penicillium ayant contaminé des yoghourts fermes
}

\author{
par \\ G. DUBOIS*
}

\section{INTRODUCTION}

Pendant l'été 1980, des yoghourts de type ferme provenant d'une laiterie de la région de Montréal ont été envahis par une moisissure vert-bleu. Nous avons été amenés à effectuer des analyses microbiologiques et macroscopiques de ce type de yoghourt. La présence de la moisissure était causée par un défaut de l'emballage au niveau du couvercle.

\section{MATERIEL ET METHODES}

Nous avons fait les mêmes analyses microbiologiques que nous avions déjà faites dans un travail antérieur [5]. Un examen microscopique à l'état frais et après coloration de Gram a été pratiqué. L'analyse a aussi consisté en une numération de la flore totale sur le milieu PCA [2], des coliformes par la méthode au bouillon lactosé bilié au vert brillant [10], des streptocoques fécaux sur milieu de Kenner et al. [7]. Les Salmonelles [8], les staphylocoques coagulase positifs [10] et les levures [6] ont été également recherchés.

L'analyse de la moisissure s'est faite au moyen d'examens macroscopiques et microscopiques. Elle a été isolée sur milieu de Sabouraud [6] et étudiée, sur milieu synthétique de Czapek [3], et sur milieu gélosé au malt [2].

* Centre de Recherches en Sciences Appliquées à l'Alimentation (CRESALA ${ }^{\mathrm{R}}$ ), Université du Québec à Montréal, C.P. 8888 Montréal H2V 1L6, Québec (Canada). 


\section{RESULTATS ET DISCUSSION}

\section{Analyse générale}

Les examens après coloration de Gram et à l'état frais ont permis la mise en évidence de bactéries Gram positives dont la présence est normale (batéries lactiques).

Le tableau 1 présente les résultats de l'analyse. La flore totale est normale et correspond à celle trouvée habituellement dans les yoghourts [1]. Le taux de coliformes et de streptocoques est très bas. Nous n'avons trouvé ni Salmonelles, ni Staphylocoques coagulase positifs, ni levures. La moisissure présente est de couleur vertbleu. Le problème vient d'un mauvais placage des couvercles aux pots de yoghourt.

\section{Analyse de la moisissure sur milieu synthétique de Czapek}

Les analyses morphologiques des caractères microscopiques et culturaux de la moisissure ont permis de faire les observations suivantes :

a) Caractères généraux

Sur milieu synthétique de Czapek, les colonies se développent en une dizaine de jours à $25^{\circ} \mathrm{C}$. Leur diamètre atteint alors 3 à $4 \mathrm{~cm}$.

\section{TABLEAU 1}

Analyse bactériologique du yoghourt ferme

\begin{tabular}{l|c}
\hline \multicolumn{1}{c|}{ Tests } & Nombre de cellules par $\mathrm{g}$ \\
\hline Flore totale & $8.7 \times 10^{8}$ \\
Coliformes & $<10$ \\
Streptocoques fécaux & $<10$ \\
Staphylocoques coagulase positive & négatif \\
Salmonelles & négatif \\
Levures & négatif \\
Moisissure (examen macroscopique) & positif \\
\end{tabular}


La couleur du bord est blanche suivie d'une zone circulaire vert-bleu. Elle devient ensuite marron-jaune et le centre est vert. Les colonies vieilles deviennent vert foncé au centre. Le dessous des colonies passe du blanc au jaune en vieillisant. Elles sont très sporulées et granuleuses. L'odeur de moisi qui se dégage est assez forte.

\section{b) Les appareils conidiens}

Les appareils conidiens ne divergent pas et les pinceaux sont asymétriques. Les chaînes de conidies sont parallèles, longues (environ $100 \mu$ ) et emmêlées.

Les conidiophores mesurent de 150 à $300 \mu$ de long par 3 à $5 \mu$ de large. Ils ont en général des branches adjointes mesurant $30 \mu$ de longueur en moyenne par $4 \mu$ de largeur. Les branches se terminent par un verticille de 3 à 5 phialides (généralement 5).

\section{c) Les conidies}

Les conidies, ont un diamètre moyen de $3 \mu$ et ont une apparence globuleuse et lisse.

\section{Analyse de la moisissure sur milieu gélosé au malt}

La moisissure se développe à $25^{\circ} \mathrm{C}$, en une dizaine de jours elle atteint un diamètre de $5 \mathrm{~cm}$. D'abord incolore, elle devient vertbleu au centre en vieillissant. Le dessous devient de couleur ocre. La moisissure ne sporule presque pas et dégage une odeur de moisi. On retrouve les mêmes caractéristiques des conidiophores sur ce milieu.

Ce penicillium étant asymétrique, nous déduisons que c'est un Asymetrica fasciculata. Comme nous retrouvons à peu près les mêmes caractères que ceux décrits par Desfleurs [4] nous pensons que la moisissure peut être Penicillium viridicatum Westling. Cette moisissure produit de l'ochratoxine qui est hautement toxique [9]. Comme cette moisissure se retrouve dans le sol et sur les graines humides, il est difficile de s'expliquer la relation de cette moisissure avec un produit laitier, sinon par le fait que l'été 1980 fut désastreux pour les cultures au Québec à cause de la pluie : la majorité des récoltes ayant été moisies.

Le problème, venant d'un mauvais placage des couvercles, heureusement limité, a été vite résolu.

\section{Rés u mé}

Une description est donnée de la moisissure Penicillium viridicatum ayant contaminé des yoghourts fermes. 


\section{Su m mary}

\section{STUDY OF PENICILLIUM HAVING CONTAMINED YOGHOURTS}

A description is given of the fungi Penicillium viridicatum having contaminated yoghourts.

Reçu pour publication en décembre 1980.

\section{Bibliographie}

[1] Accolas (J.P.), Veaux (M.), Delmas (C.), Sansoulet (O.), Grappin (R.) et PETRANSXIENE (D.) (1978). - Comptage de Streptococcus thermophilus et de Lactobacillus bulgaricus dans les yoghourts. C.R. du $20 \mathrm{e}$ Congr. Int. de Laiterie, Paris, p. 354.

[2] A.P.H.A. (1979). - Standard methods for the examination of dairy products. 14th ed W. J. Hausler Jr. editor. American Public Health Association, Washington, D.A. (U.S.A.).

[3] B.B.L. (1973). - Manual of Products and Laboratory procedures. Baltimore Biological Laboratory, 5th ed, Becton Dickinson and Company. Cockeyville, Maryland 21030 (U.S.A.).

[4] Desfleurs (M.) (1975). - Contamination de Pont-l'Evêque par un Penicil lium provenant des boîtes et cageots servant à leur emballage. Le Lait, LV, 396.

[5] Dubois (G.), Desaulniers-Therrien (F.) et Charbonneau (R.) (1980). - Rôle d'une levure dans un accident de fabrication de yoghourt brassé. Le Lait, LX, 393.

[6] F.D.A. (1976). - The Bacteriological analytical manual for foods. Foods and Drug Administration, 4th ed. A.O.A.C. Publ. Washington (U.S.A.).

[7] Kenner (B. A.), Clark (H. F.) and Kabler (P. W.) (1961). - Fecal streptococci. I. Cultivation and enumeration of streptococci in surface water. Appl. Microbiol., 9, 15.

[8] Mccoy (J. H.) (1962). - The isolation of Salmonella. J. Appl. Bacteriol., 25, 213.

[9] Moreau (Cl.) (1974). - Moisissures toxiques dans l'alimentation. 2e éd., Masson et Cie, éditeurs, Paris.

[10] Tatcher (F. S.) and Clark (D. S.) (1978). - Microorganisms in Foods. University of Toronto Press, Toronto (Canada). 